\title{
Temperature dependence of crystal field excitations in $\mathrm{CuO}$
}

\author{
Simo Huotari, ${ }^{1,2}$ Laura Simonelli, ${ }^{2}$ Christoph J. Sahle, ${ }^{1}$ Marco Moretti Sala, ${ }^{2}$ Roberto Verbeni, ${ }^{2}$ and Giulio Monaco ${ }^{2,3}$ \\ ${ }^{1}$ Department of Physics, P.O.Box 64, FI-00014 University of Helsinki, Finland \\ ${ }^{2}$ European Synchrotron Radiation Facility, B.P. 220, F-38043 Grenoble cedex, France \\ ${ }^{3}$ Physics Department, University of Trento, Via Sommarive 14, 38123 Povo (TN), Italy
}

(Dated: October 18, 2021)

\begin{abstract}
We report a study on the temperature dependence of the charge-neutral crystal field $(d d)$ excitations in cupric oxide, using nonresonant inelastic x-ray scattering (IXS) spectroscopy. Thanks to a very high energy resolution $(\Delta E=60 \mathrm{meV})$, we observe thermal effects on the $d d$ excitation spectrum fine structure between temperatures of 10-320 K. With an increasing temperature, the spectra broaden considerably. We assign the temperature dependence of the $d d$ excitations to the relatively large electron-phonon coupling.
\end{abstract}

Orbital physics in transition metal oxides (TMO's) remains in the forefront of modern physics. The complex interplay between orbital and other electron degrees of freedom results in a wide range of phenomena that pose challenges for condensed-matter physics, such as metal-insulator transitions, superconductivity, and colossal magnetoresistance $\stackrel{\underline{1}}{\underline{1}}$ Cupric oxide $\mathrm{CuO}$ (tenorite) is an antiferromagnetic insulator that has recently attracted interest as an induced multiferroic with high $T_{C}$ (Ref. 2). Unlike its periodic-table neighbors $\mathrm{NiO}$ or $\mathrm{CoO}, \mathrm{CuO}$ does not have a cubic structure with an octahedral coordination of the metal ion, but instead has a monoclinic unit cell and a slightly distorted square planar local coordination. These $\mathrm{CuO}_{4}$ units bear remarkable resemblance to the $\mathrm{CuO}_{2}$ planes in cuprate high- $T_{C}$ superconductors. The understanding of the electronic structure of $\mathrm{CuO}$ is thus an important benchmark for the quest on superconductivity. $\mathrm{CuO}$ also displays many intriguing properties such as charge-stripe ordering ${ }^{3}$ and spinphonon interaction: $\underline{4}$

The orbital excitations that take place within the $3 d$ shell of a transition metal ion, also called $d d$ or crystalfield excitations, are well known probes of the local electronic structure. They have traditionally been studied using optical absorption spectroscopies,,$\frac{5}{,}$ but electronenergy-loss spectroscopies $\underline{\underline{6}}$ and resonant IXS (RIXS) ${ }^{7}-\underline{-11}$ have been relatively recent introductions to the $d d$ excitation toolbox. Even more recently, non-resonant IXS (NRIXS) has emerged as complementary, bulk-sensitive and high-resolution tool for $d d$ excitations $\stackrel{12,13}{\underline{1} \text { Indeed, }}$ in many transition metal oxides they have been extensively studied using NRIXS $\stackrel{12,14-17}{=}$ A detailed theoretical framework has been formulated for the interpretation of non-resonant IXS for studies of $d d$ excitations via an effective operator formalism. $\underline{18}$

Only few studies have been reported on the crystalfield and charge-transfer excitations in $\mathrm{CuO}$. Opticalabsorption studies cannot probe it since the band gap 19 is smaller than the $d d$ excitation energy. Döring et $a l^{20}{ }^{20}$ studied $\mathrm{CuO}$ using RIXS by resonantly enhancing the charge-transfer excitation at the $\mathrm{Cu} K$ absorption edge $(E=8.98 \mathrm{keV})$. Ghiringhelli et al $l^{9,10}$ have studied the $d d$ excitations using RIXS at the $\mathrm{Cu} L_{3}$ absorp- tion edge $(E=930 \mathrm{eV})$. They reported $d d$ excitation spectra centered at around $\sim 2 \mathrm{eV}$. However, detailed high-resolution analyses are needed in order to fully understand the spectral characteristics and assignment of this fundamentally important compound.

$\mathrm{CuO}$ exhibits two successive magnetic transitions at

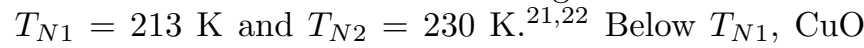
is in an antiferromagnetic commensurate collinear phase, and between the two transition temperatures in an incommensurate spiral phase. The latter phase has recently drawn interest because it has ferroelectric properties with a very high ferroelectric critical temperature $T_{C}=T_{N 2} \stackrel{2}{2}$ The band gap has also been shown to have a strong dependence of temperature, due to a relatively strong electron-phonon coupling $\stackrel{19}{\underline{19}}$ The present study aimed at the determination of the $d d$ spectra as a function of temperature, especially to see whether the two phase transitions or electron-phonon coupling have detectable influences on the spectral lineshape. While for example the electronic structure of $\mathrm{CoO}$ has been studied as a function of temperature recently, 23,24 for $\mathrm{CuO}$ temperature-dependent high-energy-resolution studies have not been reported to our knowledge.

In this article, we report high-resolution $(\Delta E=60$ meV) NRIXS spectra of $\mathrm{CuO}$ in temperatures between 10-320 K. The observable in NRIXS is the intensity of radiation scattered via an inelastic process where both momentum $\hbar \mathbf{q}$ and energy $\hbar \omega$ are transferred to the electron system. $\frac{25}{}$ In the following we assume atomic units, i.e., $\hbar=1$. The probability for scattering is quantified by the doubly differential cross section, which is related to the electron dynamic structure factor ${ }^{25}$ as

$$
\frac{\mathrm{d}^{2} \sigma}{\mathrm{d} \Omega \mathrm{d} \omega}=\left(\frac{\mathrm{d} \sigma}{\mathrm{d} \Omega}\right)_{\mathrm{Th}} S(\mathbf{q}, \omega)
$$

where $(\mathrm{d} \sigma / \mathrm{d} \Omega)_{\mathrm{Th}}$ is the Thomson scattering cross section, and $S(\mathbf{q}, \omega)$, the dynamic structure factor, contains the information on the material properties to be investigated. The same function is measured in electron energy loss spectroscopy (EELS) $\underline{\underline{6}}$ Both EELS and NRIXS have their advantages. In general, NRIXS has its strengths in being bulk sensitive, yielding also access to extreme sample environments such as high pressure, and having 
an access to high momentum transfers. The $S(\mathbf{q}, \omega)$ can be written as

$$
S(\mathbf{q}, \omega)=\sum_{F}\left|\left\langle F\left|\sum_{j} e^{i \mathbf{q} \cdot \mathbf{r}_{j}}\right| I\right\rangle\right|^{2} \delta\left(\Omega_{F}-\Omega_{I}-\omega\right),
$$

where $|I\rangle\left(\Omega_{I}\right)$ and $|F\rangle\left(\Omega_{F}\right)$ are the initial and final states (energies) of the electron system, respectively, with a summation over all electrons $j$. The dynamic structure factor is also related to the macroscopic dielectric function $\varepsilon(\mathbf{q}, \omega)$ as

$$
S(\mathbf{q}, \omega)=-\frac{n}{4 \pi e^{2}} \operatorname{Im}\left[\varepsilon^{-1}(\mathbf{q}, \omega)\right] .
$$

This equivalence is often used to relate optical spectra and dielectric screening to the results of an energy-loss experiment such as EELS or NRIXS. $\stackrel{17,26}{ }$ The theoretical framework on how NRIXS can access dipole-forbidden excitations in different systems has been laid down in, e.g., Refs. 13, $18,27,29$.

$\mathrm{CuO}$ has a monoclinic crystal structure (space group $C 2 / c$ ) (Ref. 30) with four nonequivalent $\mathrm{Cu}$ and $\mathrm{O}$ sites in the primitive unit cell $\stackrel{21,22}{ }$ The lattice parameters are $a=4.68 \AA, b=3.42 \AA, c=5.13 \AA, \alpha=\gamma=90^{\circ}$, $\beta=99.5^{\circ}$. The structure of $\mathrm{CuO}$ can be thought to consist of two different kinds of $\mathrm{CuO}_{4}$ plaquettes that are at an angle of $77.84^{\circ}$ with respect to each other. The orientation of the $\mathbf{q}$-vector with respect to the planes is thus in general an average over the two nonequivalent planes.

In the following discussion we assume $\mathrm{Cu}^{2+}$ ions in a $\mathrm{CuO}_{4}$ plaquette with a $D_{4 h}$ point group symmetry. Within the crystal field model,$\underline{31}$ the local field splits the $3 d$ energy levels to $a_{1 g}\left(d_{z^{2}}\right), b_{1 g}\left(d_{x^{2}-y^{2}}\right), b_{2 g}\left(d_{x y}\right)$, and doubly degenerate $e_{g}\left(d_{x z}\right.$ and $\left.d_{y z}\right)$. In the ground state, the hole occupies the $d_{x^{2}-y^{2}}$ orbital. More refined calculations $16,32-\underline{40}$ can be done in order to include $\mathrm{Cu}$ $\mathrm{O}$ hybridization and band structure but the crystal field model is sufficient to capture the overall energy-level picture. The $\mathrm{CuO}_{4}$ plaquettes in $\mathrm{CuO}$ are not square, but rather nerly rectangular parallelograms with side lenghts of $2.62 \AA$ and $2.90 \AA$, and exhibit two different $\mathrm{Cu}-\mathrm{O}$ distances $(1.95 \AA$ and $1.96 \AA)$. This lifts the degeneracy of the $x z$ and $y z$ orbitals, with an energy splitting that is expected to be $\sim 60 \mathrm{meV}$ (Ref. 33 ). This splitting would be expected to be resolvable with the energy resolution of the current study.

The experiment was performed at the beamline ID16 of the European Synchrotron Radiation Facility. $\underline{41}$ The incident photon beam was monochromatised using a combination of $\mathrm{Si}(111)$ premonochromator and a $\mathrm{Si}(444)$ channel-cut to a bandwidth of $40 \mathrm{meV}$. The beam was focused using a toroidal mirror to a spot size of $30 \times 100 \mu \mathrm{m}^{2}$ $(\mathrm{V} \times \mathrm{H})$ on the sample. We used a spectrometer designed for NRIXS experiments $\underline{42}$ It was equipped with six diced $\mathrm{Si}(444)$ analysers fixed at a Bragg angle of $88.5^{\circ}$, with a photon energy of $7.9 \mathrm{keV}$. The total energy resolution

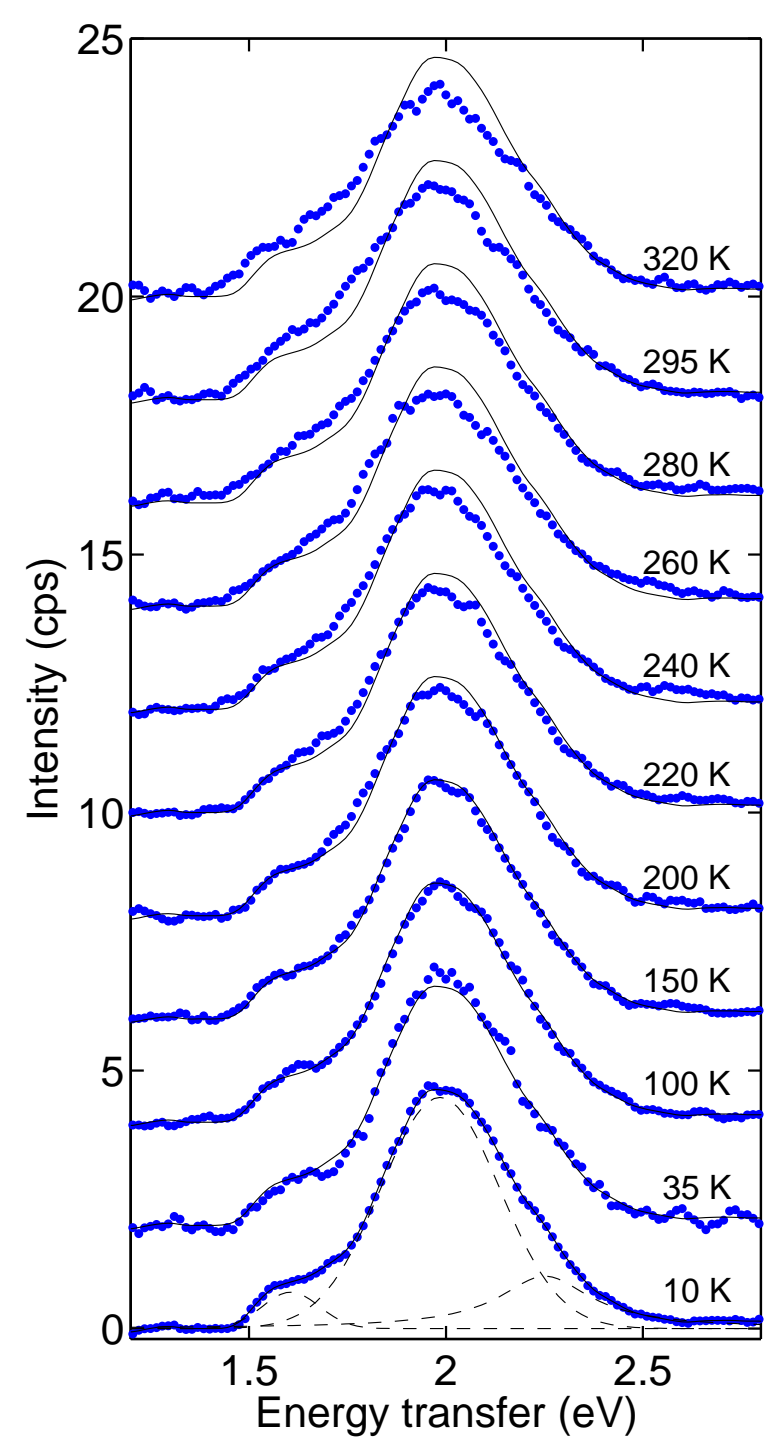

FIG. 1: (Color online) CuO d-d excitation spectra (dots) measured as a function of temperature. A smoothed version of the curve measured at $T=10 \mathrm{~K}$ (line) is shown for reference throughout. The dashed lines drawn together with the spectrum at $T=10 \mathrm{~K}$ are the result of fitting three Pearson VII functions to that spectrum.

was $60 \mathrm{meV}$. The sample temperature was controlled using a miniature He-flow cryostat. The sample was a single crystal of $\mathrm{CuO}$ (the same as used in Ref. 20). The spectra were measured at a fixed momentum transfer value $q=|\mathbf{q}|=(7.5 \pm 0.1) \AA$ with the average $\mathbf{q}$ in the direction $\mathbf{q} \| \mid$ [540]. Based on the expected angular dependence of $d d$ excitations $\frac{18}{}$, in this geometry the $x z / y z$ and $z^{2}$ peaks are expected to be excited most strongly, with the $x y$ peak to be weak. The spectra were measured in several temperatures between $10 \mathrm{~K}$ and $320 \mathrm{~K}$.

All collected spectra as a function of temperature, after subtracting a sloping background due to the quasielastic line tail, are shown in Figure 1. The spectra have been normalised to have the same area between $1-3 \mathrm{eV}$. The 
spectra can be broken into a few components: a main peak at $2 \mathrm{eV}$ and a weaker peak manifesting itself as a shoulder at $1.6 \mathrm{eV}$, and an even weaker shoulder (mainly visible at lowest temperatures) at $2.2 \mathrm{eV}$. A recent $a b$ initio calculation ${ }^{33}$ predicts the peaks to be assigned as, from lowest to highest energy, excitations from the $x^{2}-y^{2}$ orbital to the $x y, x z / y z$, and $z^{2}$ orbitals. The assignment of the peaks to specific $d$-orbital can be confirmed with either angular dependence ${ }^{8}$ or their dependence on $q$ (Ref. 18), which are subject of consequent studies. In this particular work, we concentrate on the overall temperature dependence of the spectra.

The main effect of increasing temperature from $T=10$ $\mathrm{K}$ is a clear broadening of the overall spectral shape. Due to the broadening, the low-energy shoulder seems to merge into the main peak and is nearly undetectable at room temperature. Thus, an important result is the bandwidth of the excitations: with a $60-\mathrm{meV}$ energy resolution the main peak has a width of the order of 400 meV even at $T=10 \mathrm{~K}$. This is partly due to the overlap of the $x z / y z$ and the $z^{2}$ excitation but even then the individual components have a width of about $\sim 300$ $400 \mathrm{eV}$. While in the orbital ionic picture the $3 d$ states are expected to have a very narrow line shape, when switching on the band structure the $3 d$ states gain nonnegligible bandwidth due to the electron-ion interaction and hybridization ${ }^{34,43-45}$ The observed width extrapolated to $T=0 \mathrm{~K}$ thus may reflect the width of the density of states of the occupied and unoccupied $3 d$ bands. 16 Time-dependent density functional theory that takes into account band structure, realistic transition matrix elements and local field effects could possibly explain the spectral linewidth and shape in a more detailed way. Another way of viewing this is to consider that $d d$ excitations couple to the continuum states beyond the band gap. Thus the temperature dependence could be quantified by relating the $d d$ width as a function of temperature to the thermal behavior of the band gap itself. It should be noted that the $d d$ excitations in $\mathrm{NiO}$ also have non negligible band width of the order of $200 \mathrm{meV}, \underline{49}$ even though the band gap is larger in $\mathrm{NiO}(\sim 4 \mathrm{eV}$ in comparison to $E_{g} \sim 1.35 \mathrm{eV}$ of $\mathrm{CuO}$ in room temperature).

In order to quantify the change in the shape as a function of temperature, we fitted the spectra by Pearson VII functions. ${ }^{50}$ An example of such fit in the case of $T=10 \mathrm{~K}$ is shown in Figure 11. Since the lowest and highest energy peaks are weak in this geometry, their position nor width can not be fitted very reliably. However, the determination of the width of the main peak at 2.0 $\mathrm{eV}$ can be done with a very high accuracy. The resulting fitted values for the width (full-width-at-half maximum, FWHM) of the 2.0-eV peak are shown in Fig. 2 as a function of temperature. One important result is that the peak width across different temperatures does not have a significant relation to the magnetic transitions as it does not exhibit significant changes across either transition temperature. Instead, the thermal behavior of the peak width seems to be rather smooth across the studied

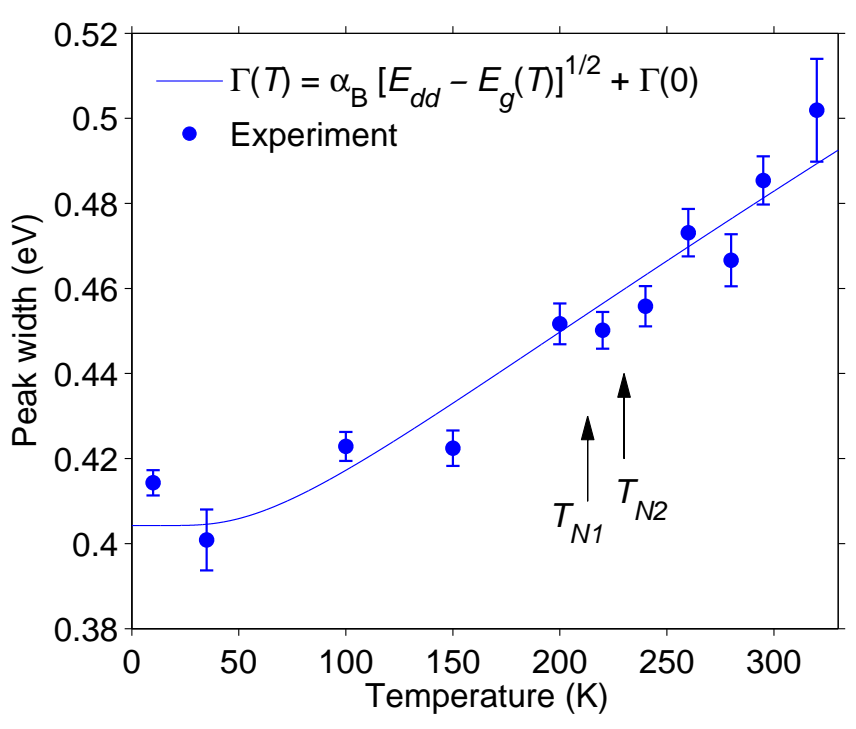

FIG. 2: The $d d$ peak width (points) compared to a fit based on the band gap width $E_{g}$ as a function of temperature (line).

temperature range.

Thus, the effect of temperature on $d d$ excitations in $\mathrm{CuO}$ is clear and easily detectable. The width of the band gap of $\mathrm{CuO}$ has been reported to vary from $E_{g}=1.55 \mathrm{eV}$ at $T=0 \mathrm{~K}$ to $1.35 \mathrm{eV}$ at $T=300 \mathrm{~K} \frac{19}{10}$ If the relatively large width of the $d d$ excitations $(\sim 400 \mathrm{meV})$ is due to interaction with continuum states owing to the presence of the band gap, the decreasing gap width with increasing temperature could explain the observed behavior. In this scenario, an increase of the density of states at, or near to, the energy of $d d$ excitations could increase the width of the $d d$ peaks. The temperature-dependence of the optical gap has been explained to be due to the large electronphonon coupling 19 Electron-phonon coupling thus seems a natural reason for the temperature-dependence of the $d d$ excitations as well.

Using a Bose-Einstein statistical factor for phonons with average energy of $k_{B} \theta$, the gap energy as a function of temperature ${ }^{19}$ can be fitted to a form ${ }^{51}$

$$
E_{\mathrm{g}}(T)=E_{\mathrm{B}}-a_{\mathrm{B}}\left[1+\frac{2}{e^{\theta / T}-1}\right]
$$

with $E_{\mathrm{B}}=1.66 \mathrm{eV}, a_{\mathrm{B}}=0.1 \mathrm{eV}$ and $\theta=196 \mathrm{~K}$. We assume a density of states above the gap of the freeelectron form $\rho(E) \propto \sqrt{E-E_{g}}$, when $E \geq E_{g}$, and $\rho(E)=0$ when $E<E_{g}$. Assuming a linear dependence of the $d d$ spectral linewidth $\Gamma$ on the density of states at the $d d$ excitation energy,

$$
\Gamma(T)=\alpha_{\mathrm{B}} \sqrt{E_{d d}-E_{g}(T)}+\Gamma(0),
$$

we get a good agreement with the experiment with $\Gamma(0)=0.0569 \mathrm{eV}$ and $\alpha_{\mathrm{B}}=0.526 \mathrm{eV}^{1 / 2}$. The resulting fit is shown in Fig. 2, Even if the observed peak here is a superposition of different $d d$ excitations, which gives 
a non-negligible contribution to $\Gamma(0)$, the temperature dependence is the most interesting result here. The fit agreement is good, yielding insight that the interaction with the continuum states could be the underlying reason for the $d d$ excitation lineshape. The temperature dependence shows a good agreement with a correspondence to the band gap variation that in turn has its underlying reasons in the electron-phonon coupling.

The $a b$ initio optical absorption spectrum in the range of $d d$ excitations in $\mathrm{NiO}$ has been calculated based on molecular dynamics simulations in finite temperature,, 52 but to our knowledge, such calculations do not exist for $\mathrm{CuO}$. A finite distribution of $\mathrm{Cu}-\mathrm{O}$ bond lengths in finite temperatures, due to thermal disorder, is expected to have an effect similar to the one observed here. This is because the $d d$ excitation energy is proportional to $a_{\mathrm{Cu}-\mathrm{O}}^{-5}$, where $a_{\mathrm{Cu}-\mathrm{O}}$ is the $\mathrm{Cu}-\mathrm{O}$ bond distance. Further, in principle, the coupling to the lattice could be possibly quantified from phonon parameters. 53,54 However, the good agreement obtained by using the phenomenological fit to the band-gap energy, already gives important insights to the coupling of the $d d$ excitations to the band gap and the electron-phonon coupling.

In conclusion, we have measured the $d d$ excitation spectra of bulk $\mathrm{CuO}$ with non-resonant IXS with high energy resolution as a function of temperature. Most importantly, the study reveals the coupling of the orbital excitations to phonons via the temperature dependence of the spectral shape. The spectral changes can be understood of being due to the interaction with the continuum states above the band gap, which in turn depends on temperature due to electron-phonon coupling. These results are important to understand the bandwidth related to the $d d$ excitations and their temperature dependence.

\section{Acknowledgments}

Beamtime was granted by the European Synchrotron Radiation Facility. Funding was provided by the Academy of Finland (Grants 1256211, 1127462, and 1259526) and University of Helsinki Research Funds (Grant 490076). We are grateful for C. Henriquet, M.C. Lagier, and the whole beamline ID16 team and support groups for expert assistance, advice, and encouragement in the experiment. We would like to thank M. W. Haverkort, M. Hakala, M. Gatti, and C. Rödl for fruitful discussions.
1 Y. Tokura and N. Nagaosa, Science 288, 462 (2000).

2 T. Kimura, Y. Sekio, H. Nakamura, T. Siegrist, and A. P. Ramirez, Nature Mater. 7, 291 (2008).

3 X. G. Zheng, C. N. Xu, Y. Tomokiyo, E. Tanaka, H. Yamada, and Y. Soejima, Phys. Rev. Lett. 85, 5170 (2000).

4 X. K. Chen, J. C. Irwin, and J. P. Franck, Phys. Rev. B 52, R13130 (1995).

${ }^{5}$ R. Rückamp, E. Benckiser, M. W. Haverkort, H. Roth, T. Lorenz, A. Freimuth, L. Jongen, A. Möller, G. Meyer, P. Reutler, et al., New J. Phys. 7, 144 (2005).

${ }^{6}$ B. Fromme, $d$-d excitations in transition metal oxides (Springer-Verlag, Berlin, 2001).

7 L. J. P. Ament, M. van Veenendaal, T. P. Devereaux, J. P. Hill, and J. van den Brink, Rev. Mod. Phys 83, 705 (2011).

8 M. Moretti Sala et al., New Journal of Physics 13, 043026 (2011).

9 G. Ghiringhelli et al., Phys. Rev. Lett. 92, 117406 (2004).

10 G. Ghiringhelli et al., Eur. Phys. J. Special Topics 169, 199 (2009).

11 J. Schlappa et al., Nature 485, 82 (2012).

12 B. C. Larson, W. Ku, J. Z. Tischler, C.-C. Lee, O. D. Restrepo, A. G. Eguiluz, P. Zschack, and K. D. Finkelstein, Phys. Rev. Lett. 99, 026401 (2007).

13 M. W. Haverkort, A. Tanaka, L. H. Tjeng, and G. A. Sawatzky, Phys. Rev. Lett. 99, 257401 (2007).

14 N. Hiraoka, H. Okamura, H. Ishii, I. Jarrige, K. D. Tsuei, and Y. Q. Cai, Eur. Phys. J. B 70, 157 (2009).

15 N. Hiraoka, M. Suzuki, K. D. Tsuei, H. Ishii, Y. Q. Cai, M. W. Haverkort, C. C. Lee, and W. Ku, EPL 96, 37007 (2011).

16 F. Iori, F. Rodolakis, M. Gatti, L. Reining, M. Upton, Y. Shvyd'ko, J.-P. Rueff, and M. Marsi, Phys. Rev. B 86,
$205132(2012)$

17 S. Huotari, J. A. Soininen, G. Vankó, G. Monaco, and V. Olevano, Phys. Rev. B 82, 064514 (2010).

18 M. van Veenendaal and M. W. Haverkort, Phys. Rev. B 77, 224107 (2008).

19 F. Marabelli, G. B. Parravicini, and F. Salghetti-Drioli, Phys. Rev. B 52, 1433 (1995).

20 G. Döring et al., Phys. Rev. B 70, 085115 (2004).

21 J. B. Forsynth, P. J. Brown, and B. M. Wanklyn, J. Phys. C: Solid State Phys. 21, 2917 (1988).

22 B. X. Yang et al., Phys. Rev. B 39, 4343 (1989).

23 R. Kurian, M. M. van Schooneveld, N. Zoltán, G. Vankó, and F. M. F. de Groot, J. Phys. Chem. C 117, 2976 (2013).

${ }^{24}$ L. A. Wray, J. Li, Z. Q. Qiu, J. Wen, Z. Xu, G. Gu, S.-W. Huang, E. Arenholz, W. Yang, Z. Hussain, et al., Phys. Rev. B 88, 035105 (2013).

25 W. Schülke, Electron Dynamics by Inelastic X-Ray Scattering (Oxford University Press, Oxford, 2007).

${ }^{26}$ H.-C. Weissker, J. Serrano, F. Bruneval, F. Sottile, S. Huotari, G. Monaco, M. Krisch, V. Olevano, and L. Reining, Phys. Rev. Lett. 97, 237602 (2006).

27 J. A. Soininen, A. L. Ankudinov, and J. J. Rehr, Phys. Rev. B 72, 045136 (2005).

28 R. Caciuffo et al., Phys. Rev. B 81, 195104 (2010).

29 R. A. Gordon, G. T. Seidler, T. T. Fister, M. W. Haverkort, G. A. Sawatzky, A. Tanaka, and T. K. Sham, EPL 81, 26004 (2008).

30 S. Åsbrink and L.-J. Norrby, Acta Crystallogr. Sect. B: Struct. Crystallogr. Cryst. Chem. (1970).

31 B. N. Figgis and M. A. Hitchman, Ligand field theory and its applications (Wiley-VCH, 2000).

${ }^{32}$ H. Eskes, L. H. Tjeng, and G. A. Sawatzky, Phys. Rev. B 
42, 288 (1990).

33 H.-Y. Huang, N. A. Bogdanov, L. Siurakshina, P. Fulde, J. van der Brink, and L. Hozoi, Phys. Rev. B 84, 235125 (2011).

34 S. Lany, Phys. Rev. B 87, 085112 (2013).

35 C. Rödl, F. Fuchs, J. Furthmüller, and F. Bechstedt, Phys. Rev. B 77, 184408 (2008).

36 C. Rödl, F. Fuchs, J. Furthmüller, and F. Bechstedt, Phys. Rev. B 79, 235114 (2009).

37 C. Rödl and F. Bechstedt, Phys. Rev. B 86, 235122 (2012).

38 A. Schrön, C. Rödl, and F. Bechstedt, Phys. Rev. B 86, 115134 (2012).

39 M. Takahashi and J. Igarashi, Phys. Rev. B 56, 12818 (1997).

40 M. W. Haverkort, M. Zwierzycki, and O. K. Andersen, Phys. Rev. B 85, 165113 (2012).

41 In 2013, The IXS beamline ID16 was replaced by a new upgraded beamline ID20 of the European Synchrotron Radiation Laboratory.

42 R. Verbeni, T. Pylkkänen, S. Huotari, L. Simonelli, G. Vankó, K. Martel, C. Henriquet, and G. Monaco, J. Synchrotron Radiat. 16, 469 (2009).

43 W. Y. Ching, Y.-N. Xu, and K. W. Wong, Phys. Rev. B 40, 7684 (1989).
44 D. Wu, Q. Zhang, and M. Tao, Phys. Rev. B 73, 235206 (2006).

45 M. Heinemann, B. Eifert, and C. Heiliger, Phys. Rev. B 87, 115111 (2013).

46 I. G. Gurtubay, J. M. Pitarke, W. Ku, A. G. Eguiluz, B. C. Larson, J. Tischler, P. Zschack, and K. D. Finkelstein, Phys. Rev. B 72, 125117 (2005).

47 S. Huotari, C. Sternemann, M. C. Troparevsky, A. G. Eguiluz, M. Volmer, H. Sternemann, H. Müller, G. Monaco, and W. Schülke, Phys. Rev. B 80, 155107 (2009).

48 A. V. Kozhevnikov, M. C. Troparevsky, T. C. Schulthess, A. G. Eguiluz, T. Pylkkänen, L. Paolasini, P. A. Metcalf, G. Monaco, and S. Huotari, to be published.

49 S. Huotari et al., to be published.

50 H. J. Wang and J. Zhou, J. Appl. Cryst. 38, 830 (2005).

51 P. Lautenschlager, M. Garriga, L. Viña, and M. Cardona, Phys. Rev. B 36, 1987 (1987).

52 A. Domingo, A. Rodríguez-Fortea, M. Swart, C. de Graaf, and R. Broer, Phys. Rev. B 85, 155143 (2012).

53 A. B. Kuz'menko et al., Phys. Rev. B 63, 094303 (2001).

54 C. C. Homes, M. Ziaei, B. P. Clayman, J. C. Irwin, and J. P. Franck, Phys. Rev. B 51, 3140 (1995). 